\title{
PRODUCTIVIDAD DE LA TIERRA AGRÍCOLA EN EL SUDOESTE BONAERENSE1
}

\author{
Marta Susana Picardi* \\ Alicia Giacchero*
}

enviado: noviembre 2014 - aceptado: agosto 2015

\begin{abstract}
Resumen
El objetivo del trabajo es analizar la evolución de la productividad de la tierra agrícola en el Sudoeste Bonaerense (SOB) durante el período 1970-2010 y hacer una comparación con el mismo indicador a nivel país, que usa índices Divisia en su versión discreta (Índice Törnqvist - Theil). Debido a la falta de información completa para trabajar con Productividad Total de Factores, en esta aproximación al tema se calcula la Productividad de la Tierra Agrícola. Los resultados muestran una tendencia levemente decreciente en la productividad de la tierra agrícola regional a la inversa de lo sucedido a nivel nacional.
\end{abstract}

Código JEL: R11, Q19

Palabras clave: productividad de la tierra agrícola, sudoeste bonaerense, comparación región - nación

* Instituto de Investigaciones Económicas y Sociales del Sur (CONICET-UNS)- Departamento de Economía - Universidad Nacional del Sur Bahía Blanca - Argentina.

E-Mail: spicardi@criba.edu.ar, aliciagiacchero@gmail.com

1 Trabajo desarrollado en el marco del PGI 24/E086 y PGI-TIR 24/TE07 de la Secretaría de Ciencia y Tecnología de la UNS. Una versión preliminar de este trabajo fue presentada en la Reunión Anual de la AAEP 2012. Las autoras agradecen la colaboración de la Cra. Marianella De Battista y el Ing. Pascual Ciccioli por el trabajo de recolección, sistematización y procesamiento inicial de los datos. También agradecen la posibilidad de avance al Dr. Lema que realizó la observación en la exposición de la versión preliminar de este trabajo en la XLVI reunión de la AAEP 


\begin{abstract}
The objective of this paper is the analysis of the evolution of agricultural land productivity in the southwestern region of Buenos Aires (SOB) during the period 1970-2010, as well as a comparison with the same indicator at country level, that uses Divisia indices in its discrete version (Index Tórnqvist - Theil). In this version only Agricultural Land Productivity could be obtained because of the lack of complete information needed in order to calculate Total Factors Productivity. The results at regional level show a slightly decreasing trend in productivity of regional agricultural land.
\end{abstract}

JEL Code: R11, Q19

Keywords: productivity of agricultural land, southwest of Buenos Aires, comparison region - nation.

\title{
INTRODUCCIÓN
}

El setenta y cinco por ciento (75\%) del territorio de la provincia de Buenos Aires tiene condiciones climáticas y de suelos que le dan ventajas comparativas para la producción primaria. La pampa húmeda y subhúmeda-húmeda tiene a las tierras agrícolas y ganaderas con uno de los mayores potenciales productivos del mundo.

Es habitual observar cómo los diseños de políticas de crédito, fomento y promoción de distintas actividades primarias, tanto de la Provincia como de la Nación, han respondido en general a las condiciones de retorno de inversión que presenta esa zona geográfica. Sin embargo, el veinticinco $(25 \%)$ restante del territorio, localizado en el Sudoeste Bonaerense (SOB) no es pampa húmeda, y forma parte de las regiones semiárida, árida y subhúmeda-seca del país con características climáticas y edáficas que la diferencian del resto de la provincia en cuanto a sus potencialidades y limitantes productivas primarias.

Por ello, el objetivo final de este trabajo es estimar la productividad total de factores (PFT) en el sector agrícola de la región del SOB con el fin de brindar evidencias para la gestión de políticas diferenciales que acompañen la real necesidad del sector, dado su carácter de territorio especial según la Ley 13.647. Con ese fin se estima un indicador parcial que en trabajos futuros se intentará profundizar. Aquí expondremos los resultados de la medición de la productividad de la tierra agrícola del SOB y un análisis comparativo con el mismo indicador estimado en Lema (2010) para el país (promedio agrícola nacional). En un trabajo 
futuro se espera poder calcular la productividad total de los factores y también comparar con el mismo indicador a nivel país.

Tal como se señala en los fundamentos de la Ley de creación del SOB, las políticas de fomento solo generan los cambios buscados cuando parten del reconocimiento de las potencialidades y limitantes que poseen los destinatarios de las mismas. Por el contrario, si esto no se toma en cuenta, se corre el serio riesgo que esas políticas no produzcan el efecto deseado, llegando incluso en algunos casos a crear nuevas situaciones de conflicto. Por eso, la importancia de hacer este tipo de medición.

\section{MARCO CONCEPTUAL METODOLÓGICO}

El concepto de productividad en sentido estricto se refiere a la relación existente entre el output y los factores productivos y por ello el análisis adecuado de eficiencia productiva para un determinado sector requiere centrarse en la productividad total y no en el de la productividad parcial, aunque la estimación parcial también puede hacerse.

La PTF no es más que el cálculo de un cociente entre producción y factores. Cualquier factor no considerado en el cálculo puede afectar la medición de la productividad. Por ejemplo la mejora genética incorporada en semillas o las mejoras en la gestión de las empresas que son dificultosas de medir pueden dar lugar a aumentos de la PTF. En ese sentido la evolución de la PTF es una excelente medida de las mejoras que típicamente implican mejor calidad por unidad de factor, mejor organización o mejores prácticas productivas.

Un aumento en la PTF implica la adopción de tecnologías, pero esto se produce en un contexto económico asociado a un conjunto de precios relativos e instituciones que puede inducir o desalentar esa incorporación.

La diferenciación teórica entre el efecto del cambio en la cantidad de los factores y el impacto del cambio tecnológico sobre el producto que muestra la PTF, supone una interdependencia entre cambios en la tecnología y cambios en el uso de los insumos, que podría no ser la misma en el proceso productivo. Por su parte desde el punto de vista práctico también es difícil separarlos claramente ya que el cambio tecnológico viene incorporado en los insumos.

Los principales procedimientos (aunque también se están utilizando otros alternativos no paramétricos) para estimar productividad se dividen en cuatro 
metodologías fundamentales: 1. Cálculo de números índices, 2. Uso de las técnicas econométricas, 3. Procedimientos de descomposición de índices y 4. Combinación del cálculo de números índices con las técnicas econométricas.

La opción del cálculo de números índices ${ }^{2}$ es un procedimiento que ofrece algunas ventajas como son la posibilidad de poder utilizar una variada gama de productos y factores productivos, que permite una cierta adaptación a las fluctuaciones temporales de algunas variables (por ejemplo los precios) y que no requiere la estimación de funciones de producción (Rodríguez González y Expósito Díaz, 2001).

El objetivo principal de los índices de producción es medir los desplazamientos de las funciones de producción o costos. Como se derivan directamente de las funciones de producción implican la elección de un tipo de función y por lo tanto ello fija una serie de supuestos sobre la forma funcional y las elasticidades de las variables. El índice Divisia que posibilita estimar una media ponderada de las tasas de crecimiento de los componentes que lo conforman, es la mejor opción entre los distintos números índice bajo determinadas condiciones: "es un indicador robusto en el caso de funciones de producción lineal, homogéneas y dos veces diferenciables, que son menos restrictivas que las Cobb Douglas o Constant Elasticity of Sustitution (CES)" (Aguayo Lorenzo et al., 2000).

Según Aguayo Lorenzo et al. $(2000)^{3}$ el uso de este tipo de índice ${ }^{4}$ para los análisis de productividad total (por sus buenas propiedades) se justifica en

2 Los números índices están diseñados para descomponer las variaciones de los agregados de valor en sus componentes de variación total en precio y volumen. Un índice de precios puede ser expresado y calculado como un promedio ponderado de las variaciones proporcionales de los precios de un conjunto específico de bienes y servicios entre dos periodos de tiempo. Análogamente, un índice de volumen puede ser expresado y calculado como un promedio ponderado de las variaciones proporcionales de los volúmenes de un conjunto específico de bienes y servicios entre dos periodos de tiempo, por ejemplo un periodo de referencia $0 \mathrm{y}$ el periodo actual $t$. Existen muchas fórmulas de números índices que difieren unas de otras principalmente en las ponderaciones que asignan a los precios o cantidades relativos individuales y en la forma particular del promedio utilizado, ya sea aritmético, geométrico, armónico, etc.

3 Estiman PTF para España y para el sector agrícola gallego tomando en forma agregada 41 productos agrícolas y ganaderos.

4 En general, un número índice es una medida estadística que expresa los cambios registrados por una variable en el tiempo, combinando simultáneamente información característica de sus niveles y de su ritmo de avance. De esta manera facilita el análisis, ya que sus valores proporcionan directamente una medida de crecimiento y retienen las características dinámicas de las series originales. Los índices compuestos son el resultado de combinar un vector de índices elementales, de forma que sintetizan su evolución conjunta en una única magnitud. 
numerosos trabajos tales como Solow (1957), Ritcher (1966), Hulten (1973) ${ }^{5}$ y Diewert (1976). Señalan también que se calcula como índice encadenado ${ }^{6}$, que según diversos autores como Diewert $(1976)^{7}$, Ball $(1985)^{8}$ o Thritle \& Bottomley (1992) son preferidos a los directos, entre otras cuestiones porque son menos sensibles a las fluctuaciones anuales de los precios. Por su parte Bureau et al. (1990) ${ }^{9}$ resaltan que la utilización de índices encadenados especialmente en el caso de la agricultura evita que fenómenos accidentales o esporádicos puedan distorsionar el resultado final. Utilizan índices encadenados para medir la productividad en la agricultura Ball(1985), Thirtle \& Bottomley (1992) ${ }^{10}$, Olavarría, Bravo - Ureta \& Cocchi (2004) ${ }^{11}$ y Rodríguez Gonzalez \& Expósito Díaz(2001) ${ }^{12}$ entre otros.

Por su parte Lema (2010) señala que la productividad total de los factores es un indicador del cambio tecnológico que genera información importante para evaluar las fuentes del crecimiento. Sin embargo, a pesar de la importancia del

5 Señala que bajo determinadas condiciones el Índice Divisia es la mejor opción siendo perfecto en el caso de funciones translogarítmicas homogéneas.

6 Los índices compuestos que son el resultado de combinar un vector de índices elementales (que se referencian a un único producto) tales como Laspeyres, Paasche o Fisher comparan directamente dos puntos en el tiempo (actual y base). Los encadenados consideran que ese pasaje entre períodos puede fragmentarse considerando los incrementos parciales, esto es, que el encadenamiento de los índices (i.e. de las variaciones) evaluados con la frecuencia de muestreo máxima posible constituye una valoración más apropiada del cambio realizado desde 0 hasta $t$ con lo que intuitivamente se intenta reducir el envejecimiento de la base.

7 Según Rodríguez González, Expósito Díaz (2001) demuestra que los índices de productividad se derivan directamente de las funciones de producción y que ello implica la elección de un tipo de función de producción y la aceptación de una serie de supuestos sobre la forma funcional y las elasticidades de las variables.

8 Realiza una estimación para el sector en USA, período 1948-1979 y utiliza para el índice de insumos trabajo y capital (incluye al factor tierra como parte del capital) y agrega insumos intermedios (energía, agroquímicos y piensos y semillas).

9 Aplican PTF para Francia e Italia durante el período 1967 - 1987. Por su parte Mergor (1993) estudia la situación para la agricultura griega y encuentra también que el crecimiento del sector se ralentiza después de su incorporación a la CEE.

10 Thritle y Bottomley (1992) hacen una estimación para el período 1967 - 1990 para UK con el fin de actualizar las estimaciones disponibles y señalan además que se realizaron esfuerzos por unificar metodologías para hacer comparaciones internacionales. Mencionan que en Hoque y Thirtle (1991) hay resultados preliminares de comparación de PTF para USA y UK.

11 Estiman PTF en la agricultura chilena, período 1961-1999, utilizando Índices Törnqvist.

12 Rodríguez González, Expósito Díaz (2001) realizan una estimación de PTF para el sector agrario español que permite actualizar los trabajos de Fernández Herruzo (1996) y de Alfranca (1995) y contrastar a partir de esto el impacto de la adhesión de España a la CEE sobre la productividad. Hacen estimación para el país pero también a nivel provincial y ven la evolución dispar que ha habido. Trabajan con datos de agricultura y ganadería. 
estudio de estos factores que determinan el crecimiento y la productividad del sector agropecuario, en Argentina tiene pocos antecedentes entre los que se puede citar a Ballesteros $(1958)^{13}$, Díaz Alejandro $(1975)^{14}$, Elías $(1985)^{15}$, Lanteri $(1994)^{16}$ y, Lema \& Brescia $(2001)^{17}$.

Se considera que existe avance tecnológico cuando la retribución a los factores de producción no agota el producto y por lo tanto surge un remanente no explicado por estos. La magnitud de esa diferencia estima el crecimiento de la productividad que se mide calculando el cambio total del producto y descontando el cambio total de los factores (diferencia entre sus tasas de crecimiento). En este sentido, como ya se dijo, hay medidas de productividad parcial y de productividad total para estimar el residuo mencionado anteriormente. La productividad parcial relaciona el volumen de producción con un único factor de producción. Esta fue la primera metodología utilizada para analizar la existencia de cambio tecnológico ${ }^{18}$, pero luego se comenzaron a estimar índices de productividad total de factores o de productividad para múltiples insumos y productos.

El índice Divisia requiere de una aproximación discreta ya que se define de forma continua en el tiempo. En este sentido la adaptación discreta más usual es la desarrollada por Törnqvist (1936) y Theil (1976) y que se denomina habitualmente como Indice Divisia-Törnqvist o IndiceTörnqvist-Theil, y se expresa como sigue:

$$
\Delta \mathrm{PTF}=\Delta \ln \mathrm{Q}-\Delta \ln \mathrm{F}
$$

Para calcular la PTF se estima un índice Törnqvist de cantidad de productos y otro de cantidad de factor o factores. Por medio de estos índices se obtienen las

13 Encontró un aumento importante del índice de producción por unidad de insumo entre 1880 y 1940 y una disminución del mismo en el período 1940 - 1952, y señala que en éste último Argentina mostró un atraso en la adopción de tecnología.

14 Analiza la evolución en el uso de los factores en el sector rural y sugiere que a partir de 1945-1949 los aumentos en la razón capital/trabajo aumenta debito a una caída en el uso de mano de obra y que ello determinó un aumento de la razón tierra/trabajo.

15 Realiza estimaciones de PTF también para Argentina y estudia las fuentes del crecimiento en la agricultura.

16 Estima igual que Reca una función de producción agregada y analiza las fuentes del crecimiento agropecuario y la innovación tecnológica entre 1950 y 1992. Toma al producto agropecuario agregado como variable dependiente versus un conjunto de insumos (trabajo, tierra, capital y fertilizantes).

17 Estiman PTF y para determinar el peso relativo de cada insumo consideran cuatro alternativas a partir de Díaz Alejandro (1975), Elías (1992) y Lanteri (1994).

18 Los datos sobre productividad total reflejan el uso de nueva tecnología pero también de una mayor adopción de tecnología existente. 
tasas de crecimiento de la producción agregada y de los factores agregados entre dos períodos de tiempo. Al calcular el índice Törnqvist de producción se pondera cada producto por su participación en el ingreso, mientras que al generar el índice Törnqvist de factores se pondera cada factor por su participación en el costo total.

El índice Törnqvist de producción es:

$$
\ln \left(Y_{t} / Y_{t-1}\right)=\frac{1}{2} \sum_{i=1}^{n}\left(\alpha_{i}+\alpha_{i-1}\right) \ln \left(\left(Y_{t} / Y_{t-1}\right)\right.
$$

Donde $Y_{i t}$ es el producto $i$-ésimo en el período $t$ y $\alpha_{i t}$ es la proporción del ingreso total generada por el producto $i$ pero valuada a precios en $t-1$ :

$$
\alpha_{i t}=\frac{P_{i t-1} Y_{i t}}{\sum_{i=1}^{n} P_{i t-1} Y_{i t}}
$$

El índice Törnqvist de factores es:

$$
\ln \left(Z_{t} / Z_{t-1}\right)=\frac{1}{2} \sum_{j=1}^{n}\left(\beta_{j t}+\beta_{j t-1}\right) \ln \left(\left(Z_{j t} / Z_{j t-1}\right)\right.
$$

Con $Z_{\mathrm{jt}}$ representando al insumo $\mathrm{j}$ en el período t y $\beta_{\mathrm{jt}}$ a la proporción del insumo $\mathrm{j}$ en el costo total en $\mathrm{t}-1$.

En este trabajo, el objetivo es medir la productividad de la tierra en la agricultura del sudoeste de la provincia de Buenos Aires para comparar con los valores estimados por Lema (2010) a nivel país. Para ello se usa el mismo instrumento con el fin de hacer compatibles los datos.

Se calcula un índice Törnqvist del valor de la producción para el periodo 1969-2009 considerando los cinco principales cultivos de cereales y oleaginosas de la región del Sudoeste Bonaerense: trigo, soja, girasol, maíz y cebada ${ }^{19}$. Los precios internos de cada producto fueron tomados de las cotizaciones de la Bolsa de Rosario (serie completa de datos de referencia de valor de los productos). En el

19 Son 4 los cultivos en el caso del trabajo de Lema (trigo, soja, maíz y girasol) mientras en éste se incluyen 5 . 
caso de la cebada cervecera ${ }^{20}$, que ha sido hasta hace muy poco tiempo un cereal de comercialización vía contrato, se toma el precio del trigo por existir una estrecha vinculación en sus cotizaciones. Los precios internos de los productos fueron deflactados utilizando el Índice de Precios Internos Mayoristas no Agropecuarios y se obtuvieron ponderadores por cultivo calculando su participación en el valor total de la producción. Ambos índices tienen como base de medición el año 1968 $=100$ de la misma forma que en el trabajo de Lema (2010).

\section{CARACTERIZACIÓN DE LA REGIÓN SOB}

La región del SOB está integrada por los partidos de Guaminí, Adolfo Alsina, Coronel Suarez, Coronel Pringles, Coronel Dorrego, Saavedra, Tornquist, Púan, Coronel Rosales, Bahía Blanca, Monte Hermoso, Villarino y Patagones. Según los últimos datos disponibles de los Censos Nacionales Agropecuarios la región comprende una superficie de 6.500 .000 hectáreas y tiene 550.000 habitantes (4\% de la población provincia), conunas8.000 explotaciones agropecuarias que ocupan alrededor del $25 \%$ del territorio de la provincia.

De acuerdo a datos de la Dirección Provincial de Estadística ${ }^{21}$, la actividad agropecuaria de la Región aporta el trece por ciento (13\%) de Producto Bruto Geográfico Provincial si se tiene en cuenta a Bahía Blanca, y el veintiocho por ciento (28\%) si se excluye del análisis a dicho distrito. Concentra el quince por ciento $(15 \%)$ del rodeo bovino de la Provincia, y es esencialmente una zona ganadera con agricultura, donde el riesgo agrícola crece de norte a sur y de este a oeste.

Según consta en los Fundamentos de la Ley de creación del Sudoeste Bonaerense, en el último decenio del Siglo XX la participación de los distintos cultivos en la producción provincial fue: centeno el cuarenta y seis por ciento (46\%), avena el cuarenta por ciento (40\%), cebada el treinta y ocho por ciento $(38 \%)$, veintiocho por ciento (28\%) en el caso del trigo, veinte por ciento $(20 \%)$ en el girasol, dieciocho por ciento (18\%) en el sorgo, catorce por ciento (14\%) en el alpiste, tres por ciento ( $3 \%$ ) del maíz, y el dos por ciento ( $2 \%$ ) en el caso de la soja

Sin embargo, el desmonte indiscriminado, la agricultura en sitios inadecuados y las prácticas de laboreo agresivas han llevado a más de una explotación a

\footnotetext{
20 Hasta hace unos años el precio de la cebada cervecera se fijaba por contrato atado al precio del trigo, actualmente se estima teniendo en cuenta el FAS Argentina y el precio en Chicago.

21 Al momento del trabajo los últimos datos disponibles se refieren a 2007.
} 
magnitudes de degradación del suelo que comprometen la continuidad productiva disminuyendo seriamente los resultados económicos, que hacen casi inviables a las empresas agropecuarias en la zona o al menos ponen en alto grado de riesgo de supervivencia a las PYMES. Sin embargo, se observan cambios en el uso de la tierra, con una recuperación del interés por la ganadería ovina y nuevas alternativas productivas como la olivicultura, los cultivos de aromáticas, la intensificación de la actividad porcina, vides o bodegas boutiques que junto a otras, se adaptan de mejor manera al ambiente que muchas de las prácticas productivas tradicionales. También se agregan las posibilidades de diferenciar y certificar commodities como la carne vacuna, achicando incluso el ciclo de producción, o la segregación de trigo, transformándolos en especialities y agregando valor al tramo inicial de la cadena.

Los actuales niveles de productividad regional pueden ser incrementados con la adopción de prácticas racionales y políticas de apoyo y fomento que respondan a los ritmos productivos propios de la situación de aridez o semiaridez. En un momento en que la nación y la provincia están reviendo las políticas y estrategias para la promoción del desarrollo territorial, la diferenciación de esta vasta zona es un hecho trascendente a la hora de diseñarla. Para ello se necesita disponer de diagnósticos realistas, tal como se propone en este trabajo, que sustenten la promoción y fomento de los sistemas de producción y sus cadenas de valor como así también de radicación y permanencia de los pobladores, el apoyo a la adopción de tecnologías, la gestión de programas de educación y capacitación, de crédito, de políticas fiscales, etc.

La región del Sudoeste ha recibido a lo largo de los años políticas públicas de apoyo a la producción primaria, como también normativas que han intentado protegerla de las contingencias climáticas. Sin embargo, muchas de ellas fueron diseñadas sin haber puesto la debida atención a las potencialidades y limitantes del ambiente en el que se ponían en práctica.

Los estudios de suelo y clima, y el conocimiento que sobre ellos se ha alcanzado, permiten visualizar con mayor claridad y objetividad las posibilidades y alternativas de generación de riqueza de la actividad agropecuaria y sus cadenas de valor.

Durante el período 1991-2005 la región estuvo ininterrumpidamente en emergencia por sequía, utilizando los alcances de la Ley $\mathrm{N}^{\circ} 10.390$ y modificatorias, para hacer frente a las distintas contingencias climáticas y en muchos casos económica. ¿Por lo tanto, si existe esta situación durante un lapso de tiempo tan grande, lo descripto no responde a "emergencias climáticas" sino por el contrario a "condiciones climáticas" permanentes. 
La declaración de zona de emergencia o desastre en virtud de la Ley $\mathrm{N}^{\circ}$ 10.390 y modificatorias, otorga beneficios crediticios e impositivos. Con respecto a estos últimos, cuando se declara emergencia -con más del cincuenta por ciento $(50 \%)$ de afectación productiva-, se producen prorrogas para el pago, mientras que en situación de desastre - con más del ochenta por ciento (80\%) de afectación-, se otorgan exenciones.

En el caso de los créditos, el Banco de la Provincia de Buenos Aires otorga esperas y renovaciones de las obligaciones pendientes, unifica deudas, suspende iniciación de juicios por cobros de acreencias vencidas hasta ciento ochenta (180) días posteriores a finalizar el periodo de emergencia. Cuando la emergencia se prolonga en el tiempo, tal como ha ocurrido en la región, aparece un nuevo problema, se acumulan obligaciones tanto a nivel fiscal como crediticio que ponen en una situación más comprometida a las empresas agropecuarias. Afecta directamente a la rentabilidad de las explotaciones, que continúan implementando modelos de producción que por no estar adaptados en forma sustentable a la zona, son incapaces de generar los recursos suficientes para recomponer su economía.

La diferenciación de la región implica también apoyar firmemente la reconversión de los sistemas de producción de tal manera que se fortalezcan aquellos que estén adaptados a las características climáticas, edáficas y agronómicas, asegurando resultados económicos que permitan la permanencia de las explotaciones en esa región y con esas características.

De igual forma las políticas de fomento y apoyo no deben contemplar beneficios para actividades que no demuestren sustentabilidad, siendo esto una condición que puede variar en función de la evolución tecnológica y de las prácticas de manejo de los sistemas productivos, que deberán demostrarlo en cada caso, y sobre todo cuando se trate de actividades consideradas marginales.

Con estos criterios, la evaluación de las situaciones de emergencia tendrá una mayor precisión y equidad en su otorgamiento, ya que partirán de considerar las condiciones climáticas, edáficas y productivas permanentes y sustentables de la región.

Durante los últimos años se vienen suscitando en el Sudoeste de la Provincia de Buenos Aires cambios importantes en el sector agropecuario, relacionados a la superficie sembrada, al nivel de producción y a los rendimientos obtenidos. La Tabla 1 permite evidenciar que un análisis punta a punta de la evolución del sector no es suficientemente concluyente, por lo que se hace necesario el cálculo de los 
índices mencionados, si se pretenden mostrar los límites regionales para alcanzar la productividad media nacional.

Como se puede ver el cálculo de indicadores utilizando la estimación de datos puntuales como la de la tabal 1 no es confiable. Según se observa durante los años 90 en la región caen la produción y los rendimientos. Sin embargo, cuando se modifica el año base ambos parámetros crecen y si se amplia el período tomando en consideración la cosecha 2004/2005 las cifran cambian drásticamente.

Si se analizan los cambios sucitados en la estructura agrícola del sudoeste de la provincia de Buenos Aires para el periodo comprendido entre 1969 y $2010^{22}$, es posible observar que la participación de los cinco principales cultivos (trigo, soja, girasol, maíz y cebada) en el total de la superficie sembrada se ha modificado sustancialmente y ello puede ser la razón del salto en los rendimientos

Al comparar (figura 1) la campaña 1969/70 con la 1979-1980, se ve en el caso del trigo una caída del 5,03\% en su participación en el total del área sembrada, mientras que en el caso del maíz se produce un incremento del 8,2\%. En cuanto al girasol se observa que la participación de la oleaginosa en la estructura agrícola de la región aumentó significativamente $(239 \%)$. Con respecto a la cebada disminuye su participación un $37,3 \%$, mientras que en paralelo se incorpora a la estructura agrícola la producción de soja representando un $0,1 \%$ de la superficie total de la región.

Figura 1. Estructura agrícola campañas 69/70 y 79/80.
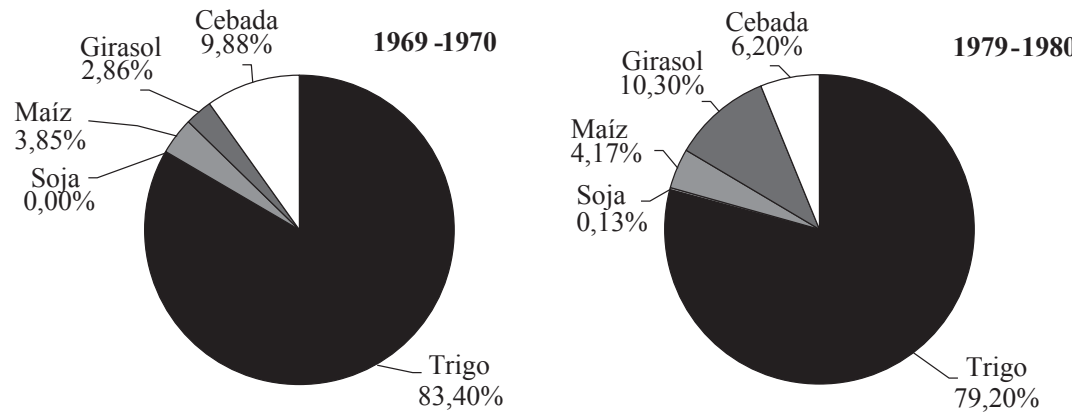

Fuente: Elaboración propia en base a datos SAGPYA.

22 Datos elaborados por la Lic. De Batista y el Ing. Ciccioli, al momento del trabajo, alumnos del Magister en Economía Agraria y Administración Rural del Departamento de Economía de la U.N.S. 
ESTUDIOS ECONOMICOS

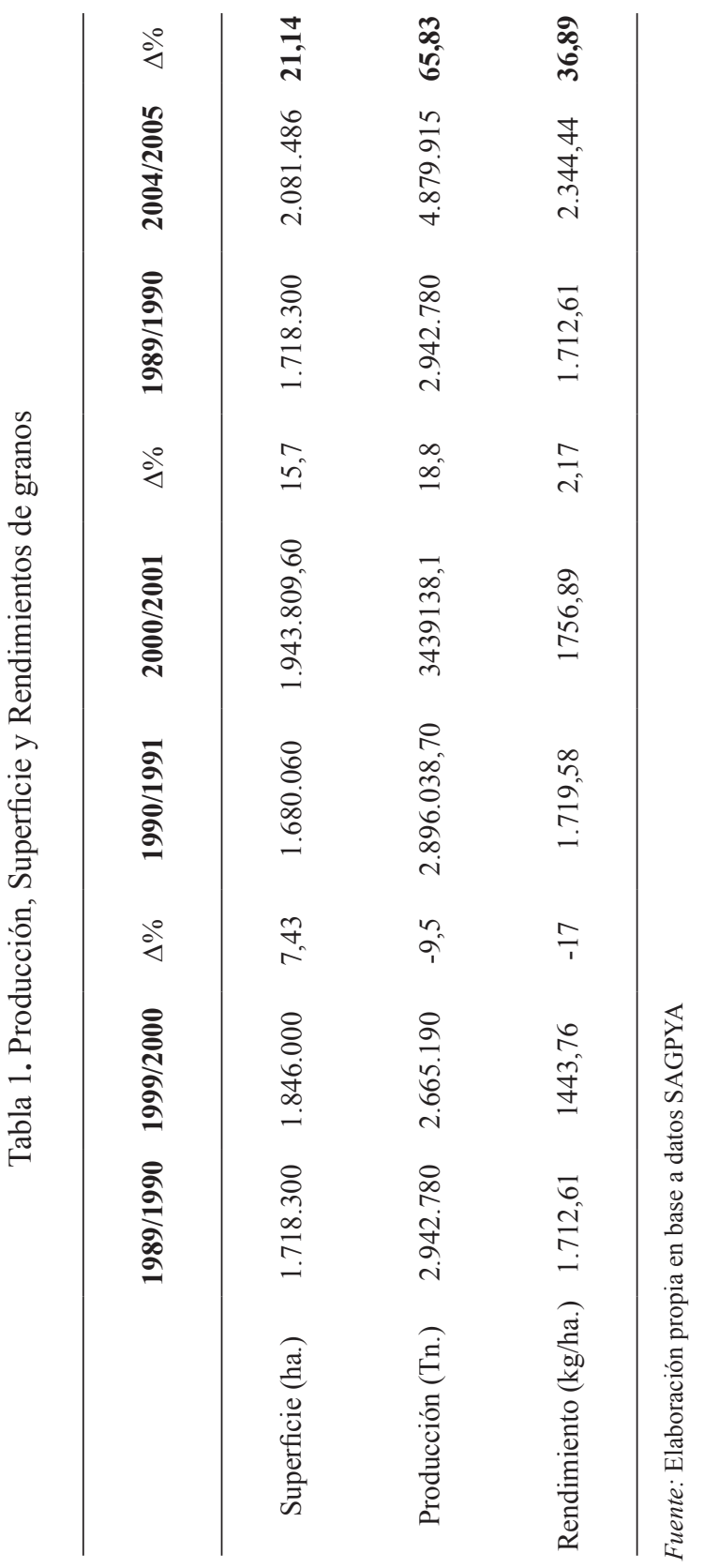


Comparando campañas del año 79/80 con $89 / 90$ el cultivo de trigo nuevamente cae en un valor cercano al $5 \%$ junto con la cebada que disminuye en un $44 \%$. El maíz aumenta un 1,5\% mientras que la soja lo hace en aproximadamente un $435 \%$. Finalmente el girasol también continúa incrementando su participación en un $58,2 \%$.

Figura 2. Estructura agrícola en las campañas 89/90 y 99/00.
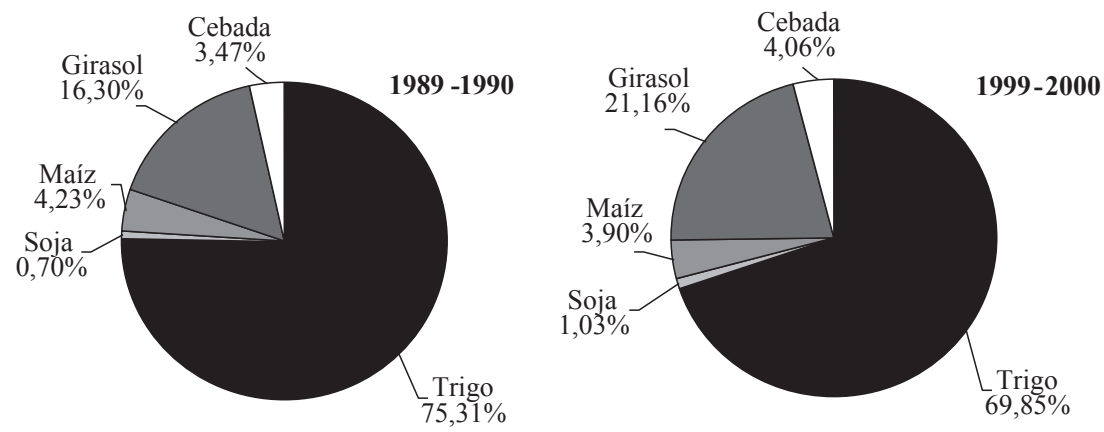

Fuente: Elaboración propia en base a datos SAGPYA.

Al analizar los cambios en la estructura agrícola en el sudoeste de la provincia de Buenos Aires comparando las campañas 1989/90-1999/2000 (Figura 2) los cultivos de trigo y maíz disminuyen su participación en un $7,3 \%$ y $7,8 \%$ respectivamente. La soja crece un $47,5 \%$ mientras que el girasol sigue el mismo comportamiento pero con un incremento del $30 \%$. Finalmente el cultivo de cebada también crece en un $17 \%$.

En la última década (Figura 3) es posible ver que el cultivo que sigue la tendencia decreciente en su participación es el trigo que pierde un $35,6 \%$ de su participación, habiendo sido el SOB la región triguera por excelencia durante muchos años en la provincia. Por otra parte se observa que la cebada aumenta un $215 \%$, mientras que la soja sigue el mismo comportamiento pero con un aumento más significativo. Pasa de un $1 \%$ al $22,7 \%$ en tanto que el girasol cae del $21,2 \%$ de la superficie total en la campaña 99/2000 al 14,7\%. 
Figura 3. Composición de la estructura agrícola en la campaña 09/10.

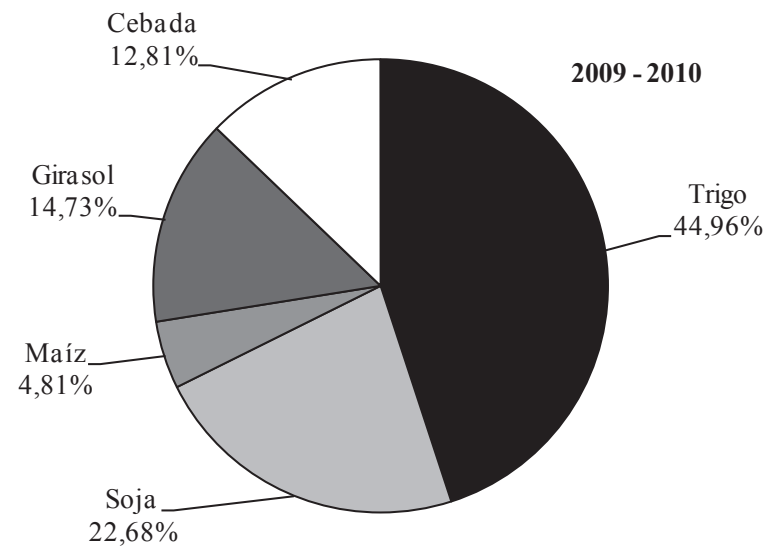

Fuente: Elaboración propia en base a datos SAGPYA.

Esta situación de cambio estructural se produce por eventos de mercado, de políticas públicas y también de problemas climáticos y de avance de la desertificación por prácticas inadecuadas de manejo. El contexto actual requiere de la atención especial en las políticas si se quisiera equidad en los impactos. Es por ello que en la sección siguiente se intenta profundizar el análisis con la consideración parcial de un único factor de características particulares: el recurso suelo cuyo potencial ha caído significativamente en los últimos años.

\section{PRODUCTIVIDAD DE LA TIERRA AGRÍCOLA (UN ANÁLISIS COMPARATIVO PAÍS - REGIÓN)}

De entre los principales procedimientos destinados a estimar la productividad, tales como el cálculo de números índices, el uso de las técnicas econométricas, los procedimientos de descomposición de índices y combinación del cálculo de números índices con las técnicas econométricas, en este trabajo se utiliza el cálculo de índices dado que se busca comparar con estimaciones realizadas a nivel nacional, que utiliza esa metodología.

El concepto de productividad en sentido estricto se refiere a la relación existente entre el output y los factores productivos. El análisis adecuado de 
eficiencia productiva para un determinado sector requiere centrarse en la productividad total y no en la productividad parcial.

Sin embargo, como se aclaró antes, la falta de datos desagregados a nivel regional nos impide en este primer abordaje del tema estimar el indicador global. Calcular la productividad parcial de la tierra agrícola es igualmente relevante, dado que posibilita tener información para la toma de decisiones en un momento en el cual el sector está reclamando políticas diferenciadas. Un índice de productividad de la tierra agrícola muestra de manera simplificada la evolución del cambio tecnológico Por lo tanto se asume que la brecha entre el índice local y el nacional, mide la deficiencia en la productividad del factor (cada hectárea de tierra rinde un mayor valor de producción a nivel nacional).

En la Figura 4 se muestra la evolución del Índice de Productividad de la Tierra Agrícola (P/T Lema) a nivel país estimado por Lema (2010) y el Índice de Productividad de la Tierra Agrícola en el SOB (P/T SO) que se estima en este trabajo. Como se observa, mientras el indicador nacional crece el regional presenta una tendencia decreciente para todo el período analizado. Ello brinda una fundamentación de lo que se dice y percibe en la región. Si se observa la Figura en la última década, primeros 10 años del Siglo XXI, la tendencia de la productividad nacional crece fuertemente mientras la regional decrece levemente.

Figura 4. Comparación Índices (Nación - SOB)

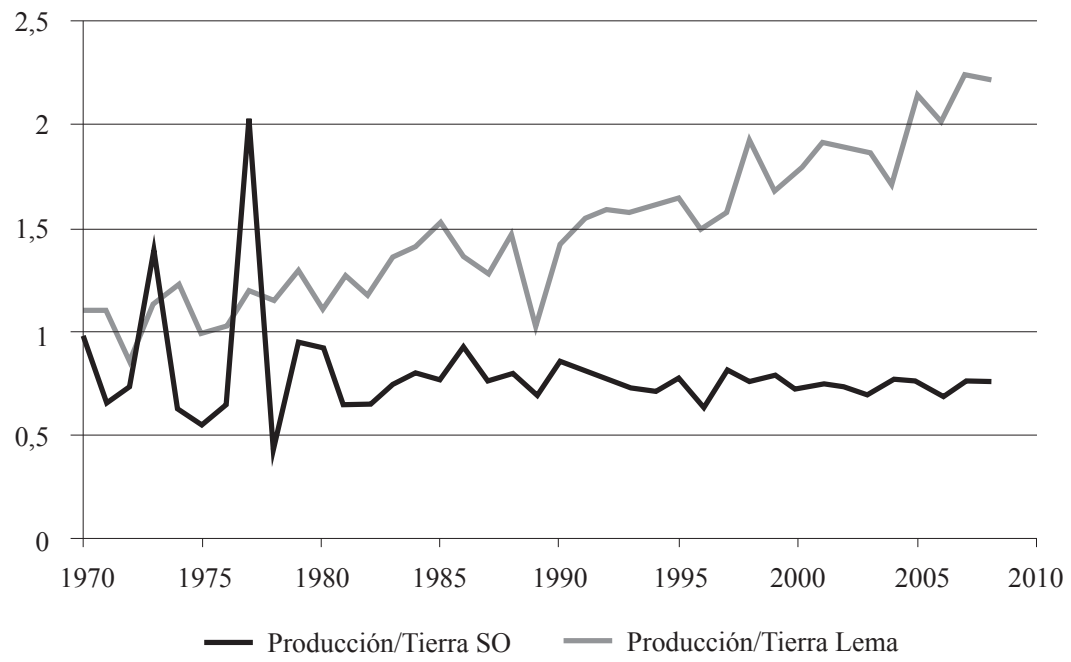

Fuente: Elaboración propia y Lema (2010) 
En la Figura $5^{23}$ se ve claramente también que mientras a nivel país hay una significativa y creciente distancia entre el Índice de Producción y el Índice Tierra evidenciando el cambio tecnológico o brecha entre unidad de producto obtenido por unidad de factor tierra utilizado, en el SOB la evolución de ambos índices presenta una leve brecha indicando que no ha habido aporte o respuesta al cambio tecnológico.

Figura 5. Índices de Producción versus Índices del Factor Tierra

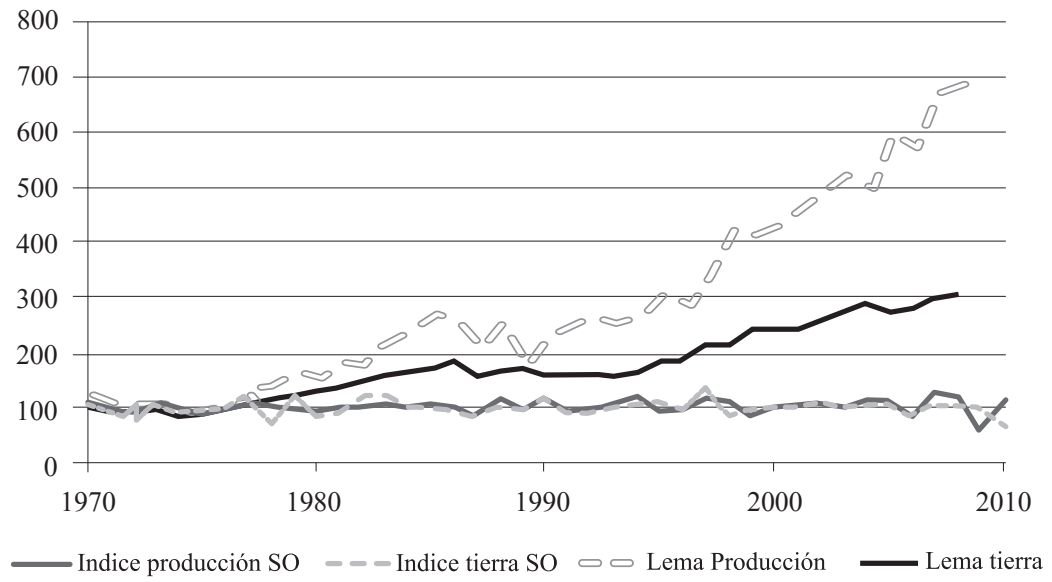

Fuente: en base a datos de elaboración propia y Lema (2010)

Cincunegui et al. (1999) señalan que la Pampa Húmeda puede ser considerada una zona homogénea en grandes rasgos pero que hay diferencias climáticas y de fertilidad que pueden afectar la fuerza del cambio tecnológico que se ha producido. Para demostrar el efecto diferencial del cambio tecnológico dividen al país según las características de las diferentes regiones (nueve en total y una de ellas es zona sudoeste: A. Alsina, Atreuco, Bahía Blanca, C. Rosales, C. Suarez, Catriló, Guaminí, Guatraché, Puán, Saavedra, Santa Rosa y Tornquist). Encuentran que en la región que está incluido el SOB es la que tiene el mínimo impacto en los rendimientos agrícolas durante el período que ellos analizan (entre los años 1982/83-1987/88 y 1992/93-1997/98).

23 La falta de coincidencia en picos y caídas que puede observarse entre ambas series pueden deberse a que los datos del SOB corresponden a campañas que toman un calendario anual de julio a julio mientras que Lema tiene datos por año calendario. 
Con el objeto de encontrar más fundamentos se analizan en la Figura 6 los rendimientos por hectárea de los cultivos tomados para el estudio en el SOB y surge que el maíz es el que mayor rendimiento ha dado y que presenta un aumento en los rindes en el tiempo a pesar de una baja participación relativa, pero que ha ido en aumento en los últimos años. Otro cultivo que ha avanzado en la zona pero para el cual no hay ventajas comparativas es la soja. El avance tecnológico con semillas genéticamente mejoras y resistentes a la sequía ha permitido su crecimiento pero no alcanza a compensar los problemas enfrentados por los trigueros que son los históricos. Siempre se la posicionó a la zona como tradicionalmente triguera y ello se corrobora además con la existencia en Bahía Blanca del puerto de Ingeniero White por el que salen fundamentalmente las exportaciones de trigo de la Argentina.

Figura 6. Evolución de los rendimientos por hectárea en el SOB

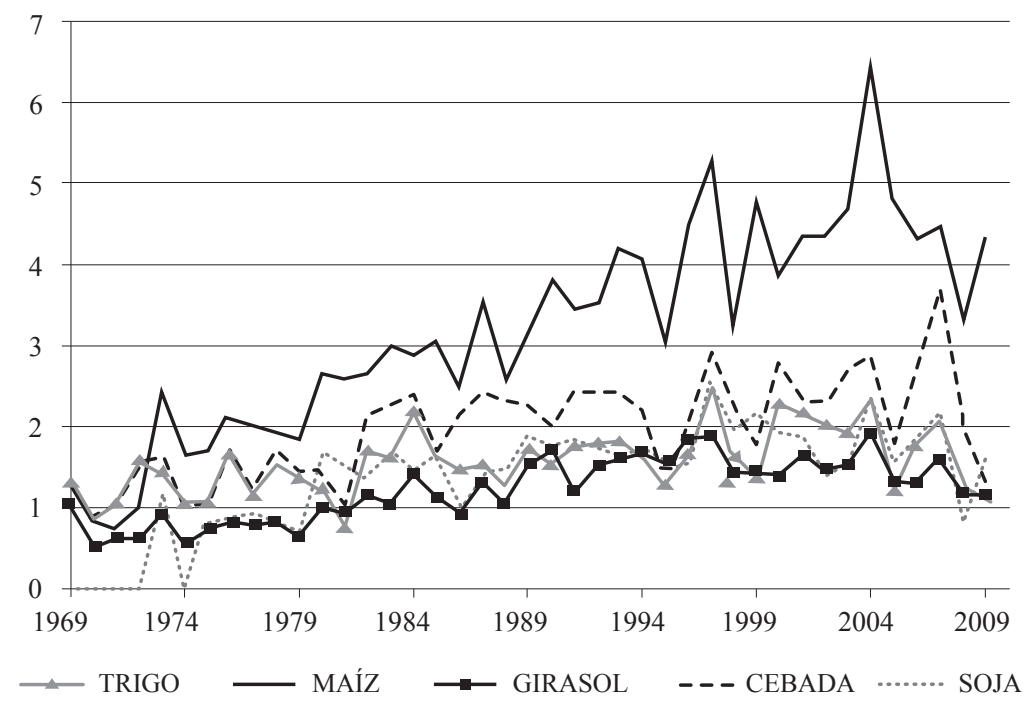

Fuente: elaboración propia en base a datos de SAGPyA.

Dado que el rendimiento de los cultivos no es decreciente se analiza la evolución de la producción total regional y como se ve en la Figura 7 si se toma la tendencia punta a punta la tasa de crecimiento es casi una constante. Esto coincide con lo que muestra el indicador Producción/Tierra SO de la Figura 5. 
Figura 7. Evolución de la producción de los cinco cultivos considerados (toneladas)

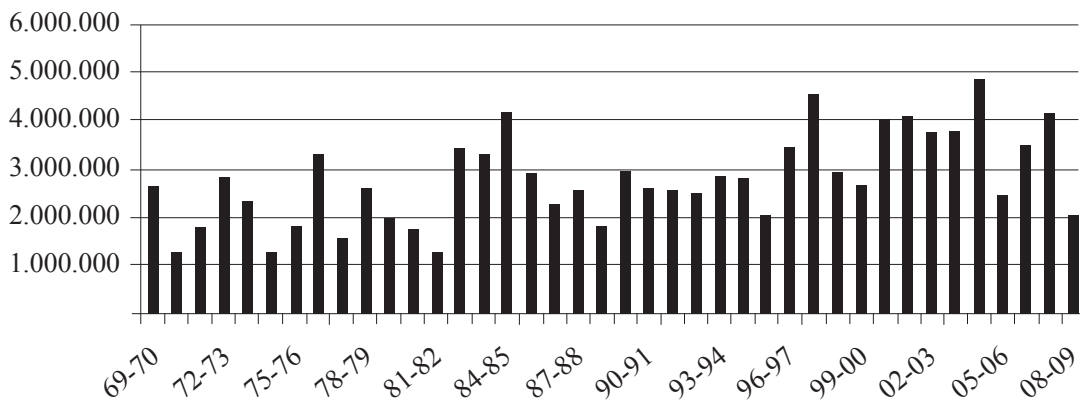

Fuente: elaboración propia en base a datos SAGPyA

Si se observa por su parte la evolución de los precios de los cereales a valores constantes del 2009 (Figura 8) se ve su deterioro en términos reales.

Figura 8. Precios reales - base 2009 (FAS teórico Puerto Rosario)

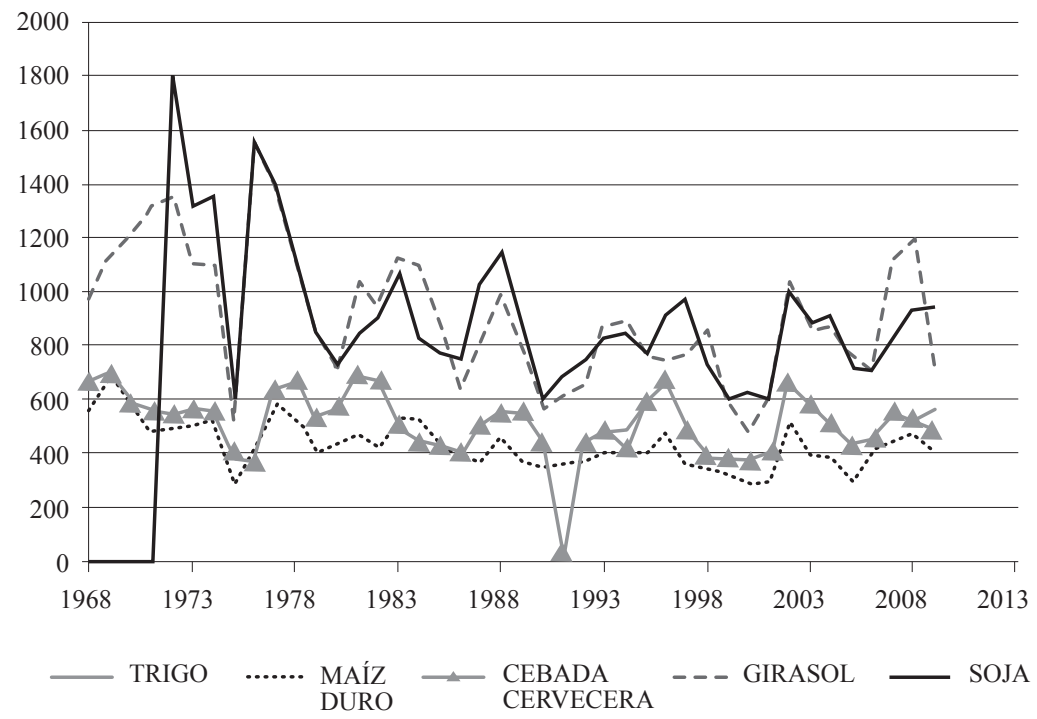

Fuente: elaboración propia precios Bolsa de Rosario, deflactor IPMNA (precio soja empieza en cero porque en 1970 no cotizaba). 
Según el análisis realizado, se evidencia la situación marginal del SOB como productora agrícola en el contexto nacional y la caída en términos reales de los precios del sector. Esto muestra que, y se constituye en una evidencia más, la región necesita de políticas y estrategias muy diferentes a las utilizadas para el sector agrícola nacional.

En esta zona marginal pareciera producirse una sinergia a partir de dos efectos: baja incorporación de tecnología o baja respuesta de la tecnología junto con una caída de precios reales que determinan una evolución, en el ingreso regional real de los productores agrícolas, con tendencia decreciente. Esto podría basarse en la alta dependencia del trigo que tiene esta región como ya se observó en la Figura 3.

Se calculó también (Figura 9) la diferencia entre las tasas de crecimiento del producto y del factor tierra para verificar el resultado (tendencia decreciente del índice - variación del índice de producción dividido la variación del índice tierra)

Figura 9. Brecha de crecimiento SOB y medición Lema

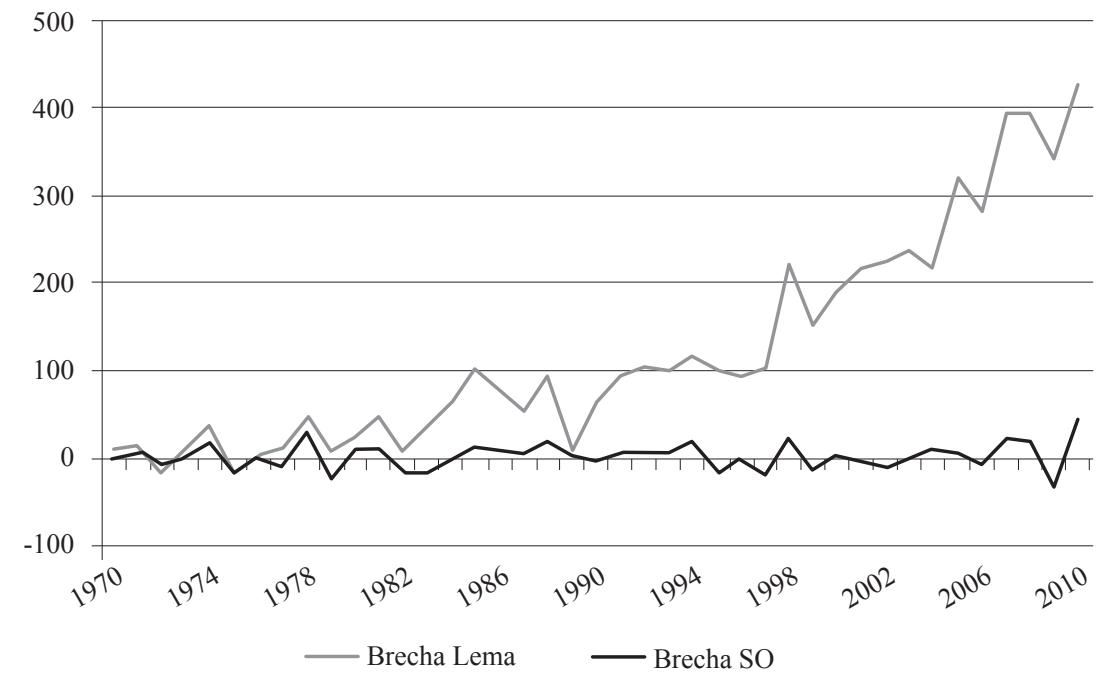

Fuente: Elaboración propia 


\section{REFLEXIONES FINALES}

Dada la situación particular del Sudoeste Bonaerense, tradicionalmente una región agrícola y ganadera que en los últimos años viene sufriendo un significativo deterioro medio ambiental, económico y por lo tanto también social, el objetivo de este trabajo es estimar la evolución de la productividad de la tierra agrícola para comparar con la evolución de la misma variable a nivel nacional.

Tal como se señala en los fundamentos de la Ley de creación del SOB, las políticas de fomento solo generan los cambios buscados cuando parten del reconocimiento de las potencialidades y limitantes que poseen los destinatarios de las mismas. Si esto no se toma en cuenta, se corre el serio riesgo que esas políticas no produzcan el efecto deseado, llegando incluso en algunos casos a crear nuevas situaciones de conflicto.

Los actuales niveles de productividad regional pueden ser incrementados con la adopción de prácticas racionales y políticas de apoyo y fomento que respondan a la potencialidad real local. En un momento en que la nación y la provincia están reviendo las políticas y estrategias para la promoción del desarrollo territorial, la diferenciación de esta vasta zona es un hecho trascendente a la hora de diseñarlas.

En el caso del sudoeste bonaerense uno de los principales cambios a realizar por parte del sector sería pasar de siembra convencional a siembra directa para lo cual deberían preparar los lotes un año antes como mínimo, razón por la cual pierden la posibilidad de cosechar en un año.

El concepto de productividad en sentido estricto se refiere a la relación existente entre el output y los factores productivos y por ello el análisis adecuado de eficiencia productiva para un determinado sector requiere centrarse en la productividad total de factores aunque la estimación parcial también puede hacerse.

Para ello y según la bibliografía, la opción del cálculo de números índice es la metodología que se usa en la mayoría de los trabajos con este objetivo. El índice Divisia en su versión Tornqvist-Theil posibilita estimar una media ponderada de tasas de crecimiento de los componentes que lo conforman (conjunto de cultivos representativos de la región) y según diversos autores son menos sensibles a las fluctuaciones de los precios. Por otro lado, el trabajo de Lema con el cual se pretende comparar usa el mismo índice. 
En este trabajo se estima el Índice de la Productividad de la Tierra Agrícola, que es una medida parcial, porque no se dispone de información completa para incorporar el total de insumos. Se considera, sin embargo, que en este caso resulta igualmente relevante como dato referencial político mostrar la evolución de este parámetro en forma comparada con el indicador a nivel nacional.

De los resultados obtenidos se ve claramente que a diferencia de lo que se observa en el indicador nacional, la productividad de la tierra en el SOB muestra una tendencia decreciente para el período 1970 - 2010 y si se restringe la serie a 1980 -2010 la misma es prácticamente constante.

Ello evidencia que la conjunción de factores climáticos, edáficos, económicos y socio culturales ha hecho que el sector agrícola de la región esté en crisis.

Dada la tendencia decreciente del índice se piensa en la posibilidad de aplicar otra metodología alternativa con el fin de interpretar el resultado tal como el indicador "Descomposición de índices" En este sentido se podrían usar trabajos que corrigen los sesgos de agregación debidos a los efectos precios y mercados en la ecuación de descomposición de dicho índice, para los casos en que los supuestos de referencia no se cumplen (Xosé Rodríguez, Pilar González, 2012), o aquellos donde las PTF negativas, utilizan la descomposición para incorporar la ineficiencia de costos, tanto técnica como de asignación (Bauer, 1988).

Lo anterior deja delimitado el avance del mismo en dos sentidos, por una parte estudiar la posibilidad y conveniencia de aplicar el método de Descomposición del Índice y hacer, una estimación de Productividad de la tierra agrícola por zonas homogéneas (grupos de partidos) de la región para determinar cuál es la que genera la caída del Indicador.

\section{REFERENCIAS BIBLIOGRAFICAS}

Aguayo Lorenzo E., Expósito Díaz, P. \& Rodríguez González, X. (2000) Productividad Total de Factores en el sector agrario gallego, 1970-1995. Análisis provincial. Universidad de Santiago de Compostela, Working Paper Serie Economic Development No. 42. Recuperado de http://www. usc.es/economet/aeeadepdf/aeeade42.pdf

Ball, V.E. (1985). Output, Input and Productivity Measurement in U.S. Agriculture, 1948-79. American Journal of Agricultural Economics, 67 (3), 475-486. 
Bauer, P (1988). Decomposing TFP growth in the presence of cost inefficiency. Federal Reserve Bank of Cleveland, Working Paper No. 8813.

Bureau, J., Butault, J., Hassan, D., Lerouvillois, P., \& Rousselle, J. (1990). Formation et Répartition des Gains de Productivité dans les Agricultures Européenes, 1967-1987. Luxembourg; Eurostat.

Cincunegui J., Vigier, H. \& Lucanera, G. Impacto regional del cambio tecnológico: La productividad granaria en Argentina entre 1984/85 y 1997/98, XXX Reunión Anual de AAEA, Bahía Blanca, Argentina.

Díaz Alejandro C.F. (1975). Ensayos sobre historia económica argentina. Buenos Aires: Amorrortu Editores.

Elías, V. J. (1992). Sources of growth. A Study of Seven Latin American Economies. International Center of Economic Growth y Fundación del Tucumán. San Francisco: ICS Press

Gallacher, Marcos (2010). The changing structure of production: Argentine agriculture 1988-2002. Económica, 56, 79-104.

Hulten, C. R. (1973). Divisa Index Numbers. Econométrica, 41 (6), 1017-1025.

Lanteri, L. N. (1994). Sources of Economic Growth and Productivity Induced Innovation and Technological Change Biases in the Agricultural Sector. Trabajo presentado en la XXIX Reunión Anual Asociación Argentina de Economía Política. La Plata, Argentina: Asociación Argentina de Economía Política. Recuperado de http://www.aaep.org.ar/anales/works/works1994/LanteriLuis.pdf

Lema D. (1998). Crecimiento y Productividad de la Agricultura Argentina 19701997. Instituto de Economía y Sociología- INTA, Documento de Trabajo. Recuperado de http://inta.gob.ar/sites/default/files/script-tmp-ventajas.pdf

Lema D. \& Bresia V. (2002). Medición del Cambio Tecnológico, la Productividad y la Eficiencia en el Sector Agropecuario. Instituto de Economía y Sociología- INTA, Documento de Trabajo.

Recuperado de http://inta.gob.ar/sites/default/files/script-tmp-medicion_ cambio_tecnologico.pdf

Lema, D. (2010), Factores de crecimiento y productividad agrícola. El rol del cambio tecnológico. En L. Reca, D. Lema, \& C. Flood (Eds.). El crecimiento de la Agricultura Argentina. Medio siglo de logros y desafios (cap. 6). Buenos Aires: Universidad de Buenos Aires, Facultad de Agronomía.

Mergos, G. (1993). Total factor productivity in agriculture: the case of Greece, 1961-1990. Trabajo presentado en el 7th Congress of the European Association of Agricultural Economists, Stresa, Italia.

Olavarría J., Bravo-Ureta B. \& Cocchi H. (2004). Productividad total de los factores en la agricultura chilena: 1961-1996. Economía Agraria y Recursos Naturales, 4 (8), 121-132. 
Rodríguez González X. \& Expósito Díaz, P. (2001). Comportamiento de la productividad total en el sector agrario español (1970-1995). Estudios de Economía Aplicada, 18 (2), 233-252.

Rodríguez, X. \& González P. (2012). Corrección de los sesgos de agregación en el convencional índice de DIVISIA para la medida de la productividad: Una aplicación para el caso de la minería española. En M. Ramos, \& F. Miranda (Eds.). Optimización-Estocástica-Recursiva-Coherente-Sistémica y sus variantes (probabilidad, econometría y estadística aplicada, Serie: Tópicos Selectos de Optimización. (pp. 67-82). España: ECORFAN.

Recuperado de http://www.ecorfan.org/series/TSO_USC_ECORFAN_ ESPA\%C3\%91A.pdf

Thirtle, C., \& Bottomley, P. (1992). Total Factor Productivity in U.K. Agriculture (1967-90). Journal of Agricultural Economics, 43 (3), 381-400.

(C) 2015 por los autores; licencia otorgada a la Revista Estudios Económicos. Este artículo es de acceso abierto y distribuido bajo los términos y condiciones de una licencia Atribución-No Comercial 3.0 Unported (CC BY-NC 3.0) de Creative Commons. Para ver una copia de esta licencia, visite http://creativecommons.org/ licenses/by-nc/3.0/ 